\title{
Neat Synthesis and Anti-oxidant Activity of $\alpha$-Hydroxyphosphonates
}

\author{
K. Uma Maheswara Rao, Ch. Syama Sundar, S. Siva Prasad, C. Radha Rani, and C. Suresh Reddy* \\ Department of Chemistry, Sri Venkateswara University, Tirupati-517 502, A.P., India. *E-mail: csrsvu@gmail.com \\ Received June 27, 2011, Accepted July 25, 2011
}

\begin{abstract}
A simple efficient and neat synthesis of $\alpha$-hydroxy phosphonates has been accomplished from aromatic/ heterocyclic aldehydes and diethyl phosphite using $\mathrm{KHSO}_{4}$ as a catalyst under solvent-free conditions at ambient temperature. These compounds are characterized by spectral and analytical data and tested for their anti-oxidant activity.
\end{abstract}

Key Words : $\alpha$-Hydroxy phosphonates, $\mathrm{KHSO}_{4}$, Anti-oxidant activity

\section{Introduction}

Oxidation reactions can produce free radicals during metabolism, which start chain reactions and damage cells. Antioxidants terminate these chain reactions by removing free radical intermediates, and also inhibit other oxidation reactions.

In recent years, a detailed mechanism of the antioxidant action of organophosphorus compounds and their relationships between chemical structure and antioxidant activity has been comprehensively studied. Phosphites and phosphonates depending on their structure act as both primary and secondary antioxidants. ${ }^{1,2}$ Reactive oxygen species (ROS) produced by univalent reduction of dioxygen to superoxide anion which in turn disproportionate to $\mathrm{H}_{2} \mathrm{O}_{2}$ and $\mathrm{O}_{2}$ spontaneously play a major role in the inflammatory process in rheumatoid arthritis (RA) and contribute to the destruction of cartilage and bone. ${ }^{3,4}$ The most important ROS implicated in inflammatory tissue injury are superoxide radical $\left(\mathrm{O}_{2}^{-}\right)$, hydrogen peroxide $\left(\mathrm{H}_{2} \mathrm{O}_{2}\right)$, hydroxyl radical $\left(\mathrm{HO}^{-}\right)$, and hypochlorous acid ( $\mathrm{HOCl})$. In the inflammed joint, these ROS are produced by macrophages, neutrophils, and chondrocytes. ${ }^{5}$ The inflammed rheumatoid joint also undergoes a hypoxia-reperfusion cycle, which results in ROS generation. ${ }^{6}$ The antioxidants may have a therapeutic role in rheumatoid arthritis by suppressing the inflammation.

The aim of this study was to investigate the in vitro antioxidant profile of different $\alpha$-hydroxy phosphonates, which, the best of our knowledge has not yet been systematically studied. In recent times, $\alpha$-hydroxyphosphonates are drawing increased attention due to their enzyme inhibitory bioactivity toward rennin, ${ }^{7}$ enolpyruvylshikimate3-phosphate synthase, ${ }^{8}$ farnesyl protein transferase, ${ }^{9}$ human immunodeficiency virus protease and polymerase. ${ }^{10}$ They also have anti-virus ${ }^{11}$ and anti-cancer activity. ${ }^{12}$

The available methods for the synthesis of $\alpha$-hydroxyphosphonates involving the nucleophilic addition of di or trialkylphosphite to different carbonyl compounds in the presence of various catalysts, such as, enzymatics, ${ }^{13}$ alkaloids, ${ }^{14}$ phosphoric acids, ${ }^{15}$ Lewis acids, ${ }^{16}$ alumina, ${ }^{17 \mathrm{a}}$ SALALEN, ${ }^{17 \mathrm{~b}}$ SALEN, ${ }^{18 \mathrm{a}}$ SALCYEN, ${ }^{18 \mathrm{~b}}$ BINOL, ${ }^{19}$ alumina/potassium fluoride, ${ }^{20} \mathrm{NH}_{4} \mathrm{VO}_{3}{ }^{21}$ and polymer/solid supported base ${ }^{22}$ were tried to improve the yields. But none of these is satisfactory. Hence, there is still a need to search for better catalysts with regards to their handling and economic viability.

Recently the commonly available $\mathrm{KHSO}_{4}$ is used as a catalyst instead of Lewis acids. ${ }^{23}$ In continuation of our interest to develop efficient synthetic routes for biologically active organophosphorus compounds using green chemical techniques for organic synthesis, ${ }^{24}$ we successfully used $\mathrm{KHSO}_{4}$ as a catalyst instead of Lewis acids for the synthesis of $\alpha$-hydroxyphosphonates under a neat condition. This catalyst is inexpensive, mild, and does not require the maintenance of anhydrous conditions. There have been no reports on the synthesis of $\alpha$-hydroxyphosphonates using $\mathrm{KHSO}_{4}$ as catalyst.

\section{Experimental}

General. NMR spectra were recorded on a Bruker instrument at $400 \mathrm{MHz}$ for ${ }^{1} \mathrm{H} \mathrm{NMR}, 100 \mathrm{MHz}$ for ${ }^{13} \mathrm{C} \mathrm{NMR}$, and 161.3 $\mathrm{MHz}$ for ${ }^{31} \mathrm{P} \mathrm{NMR}$ in $\mathrm{CDCl}_{3}$ solution, using TMS as internal and $85 \% \mathrm{H}_{3} \mathrm{PO}_{4}$ as external standard, respectively. Positive chemical shifts downfield from that of external $85 \% \mathrm{H}_{3} \mathrm{PO}_{4}$ for ${ }^{31} \mathrm{P}$ NMR spectra. Chemical shifts $(\delta)$ are indicated in ppm and coupling constants $(J)$ in Hz. Mass spectra were recorded on ESI-MS mass spectrometer and IR spectra were measured on Perkin-Elmer FT-IR 240-c spectrophotometer using $\mathrm{KBr}$ optics. Elemental analyses were performed on a Thermo Finnigan Instrument. mp were determined in open capillaries and are uncorrected. All reagents were purchased from Sigma Aldrich and were used without further purification.

General Procedure for the Synthesis of $\alpha$-Hydroxyphosphonate Derivatives. A mixture of aldehyde ( $1 \mathrm{mmol})$, diethyl phosphite $(1.5 \mathrm{mmol})$, and $\mathrm{KHSO}_{4}(20 \mathrm{~mol} \%)$ under a neat condition was stirred at rt. After completion of the reaction as indicated by TLC, the contents were poured into $\mathrm{H}_{2} \mathrm{O}$ and extracted with EtOAc. The extract was dried with anh. $\mathrm{Na}_{2} \mathrm{SO}_{4}$ and evaporated under vacuum. The crude residue was chromatographed and the products are isolated. 
Table 1. Synthesis of $\alpha$-hydroxyphosphonate derivatives (3a-j)

Entry Compounds

Diethyl hydroxy (3-nitrophenyl)methylphosphonate (3a): Semisolid. IR (KBr): 3265 (brs, OH), $1221(\mathrm{P}=\mathrm{O}$, Phosphonate), 1009 (P-O-C). ${ }^{1} \mathrm{H}-\mathrm{NMR}\left(\mathrm{CDCl}_{3}\right):$ 7.63-7.79 $(\mathrm{m}, 4 \mathrm{Ar}-\mathrm{H}) ; 5.23\left(\mathrm{~d},{ }^{2} J_{\mathrm{P}-\mathrm{H}}=10.2 \mathrm{~Hz}, \mathrm{P}-\mathrm{C}-\underline{\mathrm{H}}\right) ; 3.93-4.08(\mathrm{~m}$, $\left.4 \mathrm{H}, \mathrm{P}(\mathrm{O}) \mathrm{CH}_{2} \mathrm{CH}_{3}\right) ; 1.185\left(\mathrm{t},{ }^{3} J_{\mathrm{H}-\mathrm{H}}=7.2 \mathrm{~Hz}, 6 \mathrm{H}, \mathrm{P}(\mathrm{O}) \mathrm{CH}_{2} \mathrm{CH}_{3}\right)$. ${ }^{13} \mathrm{C}-\mathrm{NMR}\left(\mathrm{CDCl}_{3}\right): 147.47(\mathrm{C}-3) ; 141.13(\mathrm{C}-1) ; 133.86(\mathrm{C}-$ 6); 129.37 (C-5); 122.36 (C-2); 121.72 (C-4); 69.05 (P-C); $52.59\left(\mathrm{~d},{ }^{2} J_{\mathrm{P}-\mathrm{C}}=7.6 \mathrm{~Hz}, \mathrm{P}-\mathrm{OCCH} \mathrm{CH}_{3}\right) ; 18.53\left(\mathrm{~d},{ }^{3} J_{\mathrm{P}-\mathrm{C}}=5.8\right.$ $\left.\mathrm{Hz}, \mathrm{P}-\mathrm{OCH}_{2}-\mathrm{CH}_{3}\right) .{ }^{31} \mathrm{P}-\mathrm{NMR}\left(\mathrm{CDCl}_{3}\right): 21.61(\mathrm{P}=\mathrm{O})$. ESIMS $m / z 289\left(100, \mathbf{M}^{+}\right)$. Anal. calcd. for $\mathrm{C}_{11} \mathrm{H}_{16} \mathrm{NO}_{6} \mathrm{P}: \mathrm{C}$, 45.68; H, 5.58. found: C, 45.65; H, 5.54.

Diethyl hydroxy (4-methoxyphenyl)methylphosphonate (3b): Solid. mp 121-123. IR (KBr): 3281 (brs, OH), 1225 ( $\mathrm{P}=\mathrm{O}$, Phosphonate), 1052 (P-O-C). ${ }^{1} \mathrm{H}-\mathrm{NMR}\left(\mathrm{CDCl}_{3}\right)$ :
7.05-7.13 (m, 4Ar-H); $4.52\left(\mathrm{~d},{ }^{2} J_{\mathrm{P}-\mathrm{H}}=10.2 \mathrm{~Hz}, \mathrm{P}-\mathrm{C}-\underline{\mathrm{H}}\right)$; 3.96-4.25 (m, 4H, $\left.\mathrm{P}(\mathrm{O}) \mathrm{C}_{2} \mathrm{CH}_{3}\right) ; 3.48(\mathrm{~s}, 3 \mathrm{H}) ; 1.189$ (t, $\left.{ }^{3} J_{\mathrm{H}-\mathrm{H}}=7.0 \mathrm{~Hz}, 6 \mathrm{H}, \mathrm{P}(\mathrm{O}) \mathrm{CH}_{2} \mathrm{CH}_{3}\right) .{ }^{13} \mathrm{C}-\mathrm{NMR}\left(\mathrm{CDCl}_{3}\right)$ : 148.5 (C-4); 139.23 (C-1); 129.36 (C-2); 128.42 (C-6); 121.73 (C-3); 121.54 (C-5); 73.4 (P-C); $56.9\left(\mathrm{~d},{ }^{2} \mathrm{~J}_{\mathrm{P}-\mathrm{C}}=7.5\right.$ $\left.\mathrm{Hz}, \mathrm{P}-\mathrm{OCH}_{2} \mathrm{CH}_{3}\right) ; 55.9\left(\mathrm{Ar}-\mathrm{OCH}_{3}\right) ; 17.8\left(\mathrm{~d},{ }^{3} \mathrm{JP}_{\mathrm{P}-\mathrm{C}}=5.8 \mathrm{~Hz}\right.$, P-OCH $\left.2-\mathrm{CH}_{3}\right) .{ }^{31} \mathrm{P}-\mathrm{NMR}\left(\mathrm{CDCl}_{3}\right): 20.24(\mathrm{P}=\mathrm{O})$. ESI-MS $m / z 274\left(76, \mathrm{M}^{+}\right), 137(100)$ Anal. calcd. for $\mathrm{C}_{12} \mathrm{H}_{19} \mathrm{O}_{5} \mathrm{P}$ : C,52.55; H, 6.98, found: C, 52.51; H, 6.95.

Diethyl hydrox (1H-imidazol-2-yl)methylphosphonate (3c): Semisolid. IR (KBr): 3323 (NH), 3245 (brs, OH), 1219 $\left(\mathrm{P}=\mathrm{O}\right.$, Phosphonate), 1026 (P-O-C). ${ }^{1} \mathrm{H}-\mathrm{NMR}\left(\mathrm{CDCl}_{3}\right): 9.38$ (s, 1H, imidazole NH); 7.32-7.39 (m, 2Ar-H); $5.12\left(\mathrm{~d},{ }^{2} J_{\mathrm{P}-\mathrm{H}}\right.$ $=10.3 \mathrm{~Hz}, \mathrm{P}-\mathrm{C}-\underline{\mathrm{H}}) ; 3.52-3.78\left(\mathrm{~m}, 4 \mathrm{H}, \mathrm{P}(\mathrm{O}) \mathrm{CH}_{2} \mathrm{CH}_{3}\right) ; 1.23$ $\left(\mathrm{t},{ }^{3} \mathrm{~J}_{\mathrm{H}-\mathrm{H}}=7.3 \mathrm{~Hz}, 6 \mathrm{H}, \mathrm{P}(\mathrm{O}) \mathrm{CH}_{2} \mathrm{CH}_{3}\right) .{ }^{13} \mathrm{C}-\mathrm{NMR}\left(\mathrm{CDCl}_{3}\right)$ : 149.3 (C-1); 125.2 (C-3); 124.6 (C-4); 72.8 (P-C); 60.1 (d, $\left.{ }^{2} J_{\mathrm{P}-\mathrm{C}}=7.6 \mathrm{~Hz}, \mathrm{P}-\mathrm{OCH}_{2} \mathrm{CH}_{3}\right) ; 15.6\left(\mathrm{~d},{ }^{3} J_{\mathrm{P}-\mathrm{C}}=5.7 \mathrm{~Hz}, \mathrm{P}-\right.$ $\left.\mathrm{OCH}_{2}-\mathrm{CH}_{3}\right) \cdot{ }^{31} \mathrm{P}-\mathrm{NMR}\left(\mathrm{CDCl}_{3}\right): 21.72(\mathrm{P}=\mathrm{O})$. ESI-MS $\mathrm{m} / \mathrm{z}$ $235\left(100, \mathrm{M}^{+}\right)$. Anal. calcd. for $\mathrm{C}_{8} \mathrm{H}_{16} \mathrm{~N}_{2} \mathrm{O}_{4} \mathrm{P}: \mathrm{C}, 40.85 ; \mathrm{H}$, 6.86. found: C, $40.82 ; \mathrm{H}, 6.81$.

Diethyl hydroxy (1H-indol-3-yl)methylphosphonate (3d): Semisolid. IR (KBr): 3356 (NH), 3259 (brs, OH), 1119 ( $\mathrm{P}=\mathrm{O}$, Phosphonate), 1005 (P-O-C). ${ }^{1} \mathrm{H}-\mathrm{NMR}\left(\mathrm{CDCl}_{3}\right)$ : 9.94 $(\mathrm{s}, 1 \mathrm{H}$, indole $\mathrm{NH}) ; 7.02-8.66(\mathrm{~m}, 5 \mathrm{Ar}-\mathrm{H}) ; 4.76\left(\mathrm{~d},{ }^{2} \mathrm{~J}_{\mathrm{P}-\mathrm{H}}=\right.$ $10.2 \mathrm{~Hz}, \mathrm{P}-\mathrm{C}-\underline{\mathrm{H}}) ; 3.61-3.77$ (m, 4H, $\left.\mathrm{P}(\mathrm{O}) \mathrm{CH}_{2} \mathrm{CH}_{3}\right) ; 1.18(\mathrm{t}$, $\left.{ }^{3} \mathrm{~J}_{\mathrm{H}-\mathrm{H}}=7.3 \mathrm{~Hz}, 6 \mathrm{H}, \mathrm{P}(\mathrm{O}) \mathrm{CH}_{2} \mathrm{CH}_{3}\right) .{ }^{13} \mathrm{C}-\mathrm{NMR}\left(\mathrm{CDCl}_{3}\right): 138.36$ (C-4); 135.52 (C-9); 121.06 (C-2); 120.64 (C-6); 119.04 (C7); 117.35 (C-8); 116.38 (C-1); 115.15 (C-5); 76.26 (P-C); $59.96\left(\mathrm{~d},{ }^{2} J_{\mathrm{P}-\mathrm{C}}=7.6 \mathrm{~Hz}, \mathrm{P}-\mathrm{OCH}_{2} \mathrm{CH}_{3}\right) ; 16.12\left(\mathrm{~d},{ }^{3} J_{\mathrm{P}-\mathrm{C}}=5.8\right.$ $\left.\mathrm{Hz}, \mathrm{P}-\mathrm{OCH}_{2}-\mathrm{CH}_{3}\right) .{ }^{31} \mathrm{P}-\mathrm{NMR}\left(\mathrm{CDCl}_{3}\right): 20.69(\mathrm{P}=\mathrm{O})$. ESIMS $m / z 283\left(\mathrm{M}^{+}\right), 146(100)$ Anal. calcd. for $\mathrm{C}_{13} \mathrm{H}_{18} \mathrm{NO}_{4} \mathrm{P}$ : C, 55.12; H, 6.41. found: C, 55.09; H, 6.38.

Diethyl [(2-benzyloxy)phenyl]Hydroxymethylphosphonate (3e): Semisolid. IR (KBr): 3257 (brs, OH), $1218(\mathrm{P}=\mathrm{O}$, Phosphonate), 1011 (P-O-C). ${ }^{1} \mathrm{H}-\mathrm{NMR}\left(\mathrm{CDCl}_{3}\right)$ : 7.32-8.43 (m, 9Ar-H); 5.06 (s, 2H); 4.94 (d, $\left.{ }^{2} J_{\mathrm{P}-\mathrm{H}}=10.0 \mathrm{~Hz}, \mathrm{P}-\mathrm{C}-\underline{\mathrm{H}}\right)$; 3.92-4.09 (m, $\left.4 \mathrm{H}, \mathrm{P}(\mathrm{O}) \mathrm{C}_{2} \mathrm{CH}_{3}\right) ; 1.27\left(\mathrm{t},{ }^{3} J_{\mathrm{H}-\mathrm{H}}=7.2 \mathrm{~Hz}, 6 \mathrm{H}\right.$, $\left.\mathrm{P}(\mathrm{O}) \mathrm{CH}_{2} \mathrm{CH}_{3}\right) .{ }^{13} \mathrm{C}-\mathrm{NMR}\left(\mathrm{CDCl}_{3}\right): 158.8(\mathrm{C}-6) ; 136.9(\mathrm{C}-$ 1'); 128.7 (C-2); 128.6 (C-4); 128.4 (C-3'\&C-5'); 128.0 (C1); 127.4 (C-4'); 126.9 (C-2'\&C-6'); 125.3 (C-3); 124.1 (C5); $78.6\left(\mathrm{O}-\mathrm{CH}_{2}-\mathrm{Ph}\right) ; 76.1(\mathrm{P}-\mathrm{C}) ; 63.0\left(\mathrm{~d},{ }^{2} J_{\mathrm{P}-\mathrm{C}}=7.5 \mathrm{~Hz}, \mathrm{P}-\right.$ $\left.\mathrm{OCH}_{2} \mathrm{CH}_{3}\right) ; 16.3\left(\mathrm{~d},{ }^{3} \mathrm{JP}_{\mathrm{P}-\mathrm{C}}=5.7 \mathrm{~Hz}, \mathrm{P}-\mathrm{OCH}_{2}-\mathrm{CH}_{3}\right) .{ }^{31} \mathrm{P}-\mathrm{NMR}$ $\left(\mathrm{CDCl}_{3}\right): 22.70(\mathrm{P}=\mathrm{O})$. ESI-MS $m / z 350\left(85, \mathrm{M}^{+}\right), 213(100)$. Anal. calcd. for $\mathrm{C}_{18} \mathrm{H}_{23} \mathrm{O}_{5} \mathrm{P}: \mathrm{C}, 61.71 ; \mathrm{H}, 6.62$. found: $\mathrm{C}$, 61.68; H, 6.58 .

Diethyl (2,6-dimethoxyphenyl)Hydroxymethylphosphonate (3f): Semisolid. IR (KBr): 3278 (brs, OH), 1208 $\left(\mathrm{P}=\mathrm{O}\right.$, Phosphonate), 1028 (P-O-C). ${ }^{1} \mathrm{H}-\mathrm{NMR}\left(\mathrm{CDCl}_{3}\right)$ : 6.817.02 (m, 3Ar-H); $4.72\left(\mathrm{~d},{ }^{2} \mathrm{~J}_{\mathrm{P}-\mathrm{H}}=10.2 \mathrm{~Hz}, \mathrm{P}-\mathrm{C}-\underline{\mathrm{H}}\right) ; 3.87-4.16$ $\left(\mathrm{m}, 4 \mathrm{H}, \mathrm{P}(\mathrm{O}) \mathrm{C}_{2} \mathrm{CH}_{3}\right) ; 3.78(\mathrm{~s}, 3 \mathrm{H}) ; 3.62(\mathrm{~s}, 3 \mathrm{H}) ; 1.33(\mathrm{t}$, $\left.{ }^{3} \mathrm{~J}_{\mathrm{H}-\mathrm{H}}=7.0 \mathrm{~Hz}, 6 \mathrm{H}, \mathrm{P}(\mathrm{O}) \mathrm{CH}_{2} \mathrm{CH}_{3}\right) .{ }^{31} \mathrm{P}-\mathrm{NMR}\left(\mathrm{CDCl}_{3}\right): 23.71$ $(\mathrm{P}=\mathrm{O})$. ESI-MS $m / z 304\left(76, \mathrm{M}^{+}\right), 167$ (100). Anal. calcd. for $\mathrm{C}_{13} \mathrm{H}_{21} \mathrm{O}_{6} \mathrm{P}: \mathrm{C}, 51.31 ; \mathrm{H}, 6.96$. found: $\mathrm{C}, 51.28 ; \mathrm{H}, 6.92$.

Diethyl (4-hydroxyphenyl)Hydroxymethylphosphonate (3g): Solid. mp 110-112. IR (KBr): 3291 (brs, OH), 3249 (brs, OH), 1205 ( $\mathrm{P}=\mathrm{O}$, Phosphonate), 1025 (P-O-C). ${ }^{1} \mathrm{H}-$ NMR $\left(\mathrm{CDCl}_{3}\right): 6.56-6.94(\mathrm{~m}, 4 \mathrm{Ar}-\mathrm{H}) ; 4.80\left(\mathrm{~d},{ }^{2} J_{\mathrm{P}-\mathrm{H}}=10.4\right.$ 


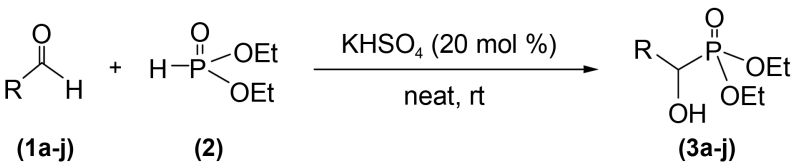

Scheme 1. Synthesis of $\alpha$-hydroxyphosphonates (3a-j).

$\mathrm{Hz}, \mathrm{P}-\mathrm{C}-\underline{\mathrm{H}}) ; 3.65-3.84\left(\mathrm{~m}, 4 \mathrm{H}, \mathrm{P}(\mathrm{O}) \mathrm{C}_{2} \mathrm{CH}_{3}\right) ; 1.13$ (t, ${ }^{3} \mathrm{~J}_{\mathrm{H}-\mathrm{H}}$ $\left.=7.2 \mathrm{~Hz}, 6 \mathrm{H}, \mathrm{P}(\mathrm{O}) \mathrm{CH}_{2} \mathrm{CH}_{3}\right) .{ }^{31} \mathrm{P}-\mathrm{NMR}\left(\mathrm{CDCl}_{3}\right): 22.63(\mathrm{P}=\mathrm{O})$. ESI-MS $m / z 260\left(100, \mathrm{M}^{+}\right)$. Anal. calcd. for $\mathrm{C}_{11} \mathrm{H}_{17} \mathrm{O} 5 \mathrm{P}: \mathrm{C}$, 50.77; H, 6.58. found: C, 50.72; H, 6.51.

Diethyl hydroxy (4-isopropylphenyl)methylphosphonate (3h): Semisolid. IR (KBr): 3273 (brs, OH), $1240(\mathrm{P}=\mathrm{O}$, Phosphonate), 1042 (P-O-C). ${ }^{1} \mathrm{H}$ NMR $\left(\mathrm{CDCl}_{3}\right)$ : 7.19-7.38 $(\mathrm{m}, 4 \mathrm{Ar}-\mathrm{H}) ; 4.94\left(\mathrm{~d},{ }^{2} J_{\mathrm{P}-\mathrm{H}}=10.4 \mathrm{~Hz}, 1 \mathrm{H}\right) ; 3.92-4.08(\mathrm{~m}, 4 \mathrm{H}$, $\left.\mathrm{P}(\mathrm{O}) \mathrm{CH}_{2} \mathrm{CH}_{3}\right) ; 2.85-2.92(\mathrm{~m}, 1 \mathrm{H}) ; 1.17-1.26(\mathrm{~m}, 6 \mathrm{H}) ; 1.165$ $\left(\mathrm{t},{ }^{3} J_{\mathrm{H}-\mathrm{H}}=7.2 \mathrm{~Hz}, 6 \mathrm{H}, \mathrm{P}(\mathrm{O}) \mathrm{CH}_{2} \mathrm{CH}_{3}\right) .{ }^{31} \mathrm{P} \mathrm{NMR}\left(\mathrm{CDCl}_{3}\right)$ : $22.83(\mathrm{P}=\mathrm{O})$. ESI-MS $m / z 286\left(100, \mathrm{M}^{+}\right)$. Anal. calcd. for $\mathrm{C}_{14} \mathrm{H}_{23} \mathrm{O}_{4} \mathrm{P}: \mathrm{C}$, 58.73; H, 8.10. found: C, 58.69; H, 8.07.

Diethyl (4-(dimethylamino)phenyl)Hydroxymethylphosphonate (3i): Semisolid. IR ( $\mathrm{KBr})$ : 3246 (brs, OH), 1237 ( $\mathrm{P}=\mathrm{O}$, Phosphonate), 1061(P-O-C). ${ }^{1} \mathrm{H}-\mathrm{NMR}\left(\mathrm{CDCl}_{3}\right)$ : 6.87-7.01 (m, 4Ar-H); 5.18 (d, $\left.{ }^{2} J_{\mathrm{P}-\mathrm{H}}=10.3 \mathrm{~Hz}, \mathrm{P}-\mathrm{C}-\underline{\mathrm{H}}\right) ; 4.02-$ $4.29\left(\mathrm{~m}, 4 \mathrm{H}, \mathrm{P}(\mathrm{O}) \mathrm{CH}_{2} \mathrm{CH}_{3}\right) ; 1.19\left(\mathrm{t},{ }^{3} \mathrm{~J}=7.3 \mathrm{~Hz}, 6 \mathrm{H}\right.$, $\left.\mathrm{P}(\mathrm{O}) \mathrm{CH}_{2} \mathrm{CH}_{3}\right) ; 2.82(\mathrm{~s}, 6 \mathrm{H}) .{ }^{31} \mathrm{P}-\mathrm{NMR}\left(\mathrm{CDCl}_{3}\right): 21.40$ $(\mathrm{P}=\mathrm{O})$. ESI-MS m/z $287\left(89, \mathrm{M}^{+}\right), 150$ (100). Anal. calcd. for $\mathrm{C}_{13} \mathrm{H}_{22} \mathrm{NO}_{4} \mathrm{P}: \mathrm{C}, 54.35 ; \mathrm{H}, 7.72$. found: $\mathrm{C}, 54.31 ; \mathrm{H}, 7.69$.

Diethyl (3,5-dimethoxyphenyl)Hydroxymethylphosphonate (3j): Solid. mp 101-103. IR (KBr): 3272 (brs, OH), $1212\left(\mathrm{P}=\mathrm{O}\right.$, Phosphonate), 1032 (P-O-C). ${ }^{1} \mathrm{H}-\mathrm{NMR}\left(\mathrm{CDCl}_{3}\right)$ : 6.78-7.07 (m, 4Ar-H); $4.87\left(\mathrm{~d},{ }^{2} J_{\mathrm{P}-\mathrm{H}}=10.2 \mathrm{~Hz}, \mathrm{P}-\mathrm{C}-\underline{\mathrm{H}}\right)$; 3.90-4.19 (m, 4H, $\left.\mathrm{P}(\mathrm{O}) \underline{\mathrm{C}}_{2} \mathrm{CH}_{3}\right) ; 3.86(\mathrm{~s}, 3 \mathrm{H}) ; 3.57$ (s, 3H); $1.28\left(\mathrm{t},{ }^{3} \mathrm{~J}=7.2 \mathrm{~Hz}, 6 \mathrm{H}, \mathrm{P}(\mathrm{O}) \mathrm{CH}_{2} \mathrm{CH}_{3}\right) .{ }^{31} \mathrm{P}-\mathrm{NMR}\left(\mathrm{CDCl}_{3}\right)$ : $22.20(\mathrm{P}=\mathrm{O})$. ESI-MS: $304\left(100, \mathrm{M}^{+}\right)$. Anal. calcd. for $\mathrm{C}_{13} \mathrm{H}_{21} \mathrm{O}_{6} \mathrm{P}$ : C 51.31, H 6.96; found: C 51.29, H 6.92.

\section{Results and Discussions}

In an initial study to optimize the experimental conditions, we carried out the reaction of 3-nitrobenzaldehyde (1), and diethyl phosphite (2) in the presence of $\mathrm{KHSO}_{4}$ with various catalytic amounts (Table 2, 10-40 mol \%) in various solvents (Table 3) at ambient temperatures. Excellent results were obtained, when the reaction was performed with $20 \mathrm{~mol} \%$ of $\mathrm{KHSO}_{4}$ under a neat condition. It was found that the amount of $\mathrm{KHSO}_{4}$ affects the yield of the product. The

Table 2. Influence of the catalyst on the synthesis of $\alpha$-hydroxyphosphonate $^{a}$

\begin{tabular}{cccc}
\hline Entry & Catalyst (mol \%) & Time (h) & Yield $^{b}(\%)$ \\
\hline 1 & $\mathrm{KHSO}_{4}(10)$ & 3.5 & 85 \\
2 & $\mathrm{KHSO}_{4}(20)$ & 2 & 91 \\
3 & $\mathrm{KHSO}_{4}(30)$ & 2.5 & 80 \\
4 & $\mathrm{KHSO}_{4}(40)$ & 2.5 & 80 \\
\hline
\end{tabular}

${ }^{a}$ Reaction of 3-nitrobezaldehyde $(1 \mathrm{mmol})$, diethyl phosphite $(1.5 \mathrm{mmol})$ under neat condition at rt. ${ }^{b}$ Isolated yield.
Table 3. Influence of the solvent on the synthesis of $\alpha$-hydroxyphosphonate $^{a}$

\begin{tabular}{cccc}
\hline Entry & Solvent & Time $(\mathrm{h})$ & Yield $^{b}(\%)$ \\
\hline 1 & Ethanol & 7 & 40 \\
2 & dioxane & 6 & 65 \\
3 & THF & 6 & 84 \\
4 & Acetonitrile & 6 & 50 \\
5 & Dichloromethane & 6 & 60 \\
6 & Toluene & 6 & 80 \\
7 & neat & 2 & 91 \\
\hline
\end{tabular}

${ }^{a}$ Reaction of 3-nitrobezaldehyde $(1 \mathrm{mmol})$, diethyl phosphite $(1.5 \mathrm{mmol})$ using $20 \mathrm{~mol} \% \mathrm{KHSO}_{4}$ at rt. ${ }^{b}$ Isolated yield.

reaction was carried out with a wide variety of aromatic and heterocyclic aldehydes bearing various substituents under optimized conditions. The prepared $\alpha$-hydroxyphosphonates (3a-j) were thoroughly characterized with spectral and elemental analysis.

IR absorptions for hydroxyl group for $\mathbf{3 a - j}$ appeared as broad signal in the region $3245-3281 \mathrm{~cm}^{-1}$ and for $\mathrm{P}=\mathrm{O}$ group absorption appeared in the region $1205-1240 \mathrm{~cm}^{-1} .^{25}$ The P $\underline{\mathrm{CH}}$ group exhibited doublet at $\delta 4.52-5.23$ due to its coupling with phosphorus. ${ }^{25}$ The carbon chemical shifts in the title compounds were observed in their expected regions. ${ }^{26}$ Their ${ }^{31} \mathrm{P}$ NMR chemical shifts were observed at $\delta$ 20.24-22.71. ESI-MS spectra gave molecular ions and diagnostic daughter ion peaks at their respective expected $m / z$ values.

Antioxidant Activity. As predicted, the $\alpha$-hydroxy phosphonates 3a-j have the ability to scavenge the DPPH radical by donating one electron. Since phosphorus has affinity towards oxygen, it can easily bind and scavenge ROS effectively. The compounds $\mathbf{3 a - j}$, containing phosphorus and oxygen atoms are more active due to the presence of nonbonded electron pairs containing hetero atoms that serve as binding sites in the bio-matrix. 3a, 3c and 3d displayed appreciable antioxidant activity. 3a showed the highest activity because of $-\mathrm{NO}_{2}$ substituent which affects the electron and hydrogen donating capacities. Since $-\mathrm{NO}_{2}$ group is highly electron withdrawing moiety, the electron density around $\mathrm{P}=\mathrm{O}$ moiety decreases and increases affinity towards oxygen derived free radicals and mobilizes ROS to be scavenged out of the living system.

DPPH Radical Scavenging Activity. The free radical scavenging activity of $\alpha$-hydroxyphosphonates against DPPH radical was performed in accordance with Choi et al. ${ }^{27} 85$ $\mu \mathrm{M}$ of DPPH was added to a medium containing different $\alpha$ hydroxyphosphonates. The medium was incubated for 30 min at room temperature. The decrease in absorbance was measured at $518 \mathrm{~nm}$. Ascorbic acid was used as standard reference to record maximal decrease in DPPH radical absorbance. The values are expressed in percentage of inhibition of DPPH radical absorbance with those of the standard control values without the title compounds (Figure 1) (ascorbic acid maximal inhibition was considered 100\% of inhibition). 


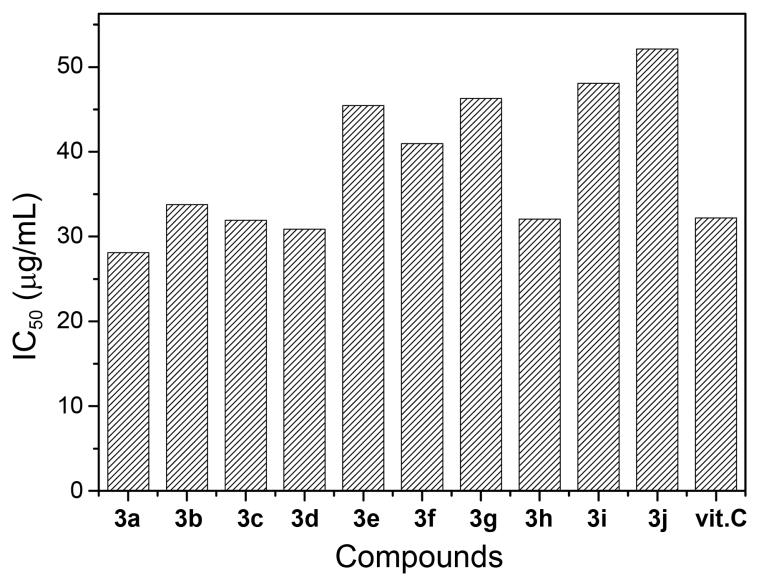

Figure 1. DPPH Radical Scavenging Activity.

$$
\text { DPPH Scavenged }(\%)=\frac{\left(A_{\text {cont }}-A_{\text {test }}\right)}{A_{\text {cont }}} \times 100
$$

Where $A_{\text {cont }}$ is the absorbance of the control reaction and $A_{\text {test }}$ is the absorbance in the presence of the sample.

In the case of $\alpha$-hydroxyphosphonates $\mathbf{3 a} \mathbf{a} \mathbf{j}$, nitro substituted compound 3a showed the highest DPPH radical scavenging activity with $\mathrm{IC}_{50}$ at $28.08 \mu \mathrm{g} / \mathrm{mL}$ when compared with others. The remaining compounds exhibited DPPH radical scavenging activity in the following order: 3d $\left(\mathrm{IC}_{50} 30.86 \mu \mathrm{g} / \mathrm{mL}\right), \mathbf{3 c}\left(\mathrm{IC}_{50} 31.91 \mu \mathrm{g} / \mathrm{mL}\right), \mathbf{3 h}\left(\mathrm{IC}_{50} 32.05\right.$ $\mu \mathrm{g} / \mathrm{mL}), \mathbf{3 b}\left(\mathrm{IC}_{50} 33.78 \mu \mathrm{g} / \mathrm{mL}\right), \mathbf{3 f}\left(\mathrm{IC}_{50} 40.98 \mu \mathrm{g} / \mathrm{mL}\right), \mathbf{3 e}$ ( $\left.\mathrm{IC}_{50} 45.45 \mu \mathrm{g} / \mathrm{mL}\right), 3 \mathrm{~g}\left(\mathrm{IC}_{50} 46.29 \mu \mathrm{g} / \mathrm{mL}\right), 3 \mathbf{3 i}\left(\mathrm{IC}_{50} 48.06\right.$ $\mu \mathrm{g} / \mathrm{mL}), 3 \mathbf{j}\left(\mathrm{IC}_{50} 52.12 \mu \mathrm{g} / \mathrm{mL}\right)$ and when compared with ascorbic acid ( $\mathrm{IC}_{50} 32.2 \mu \mathrm{g} / \mathrm{mL}$ ).

Reducing Power Assay. The reducing power of $\mathbf{3 a} \mathbf{a}-\mathbf{j}$ was determined according to the method of Oyaizu et al. ${ }^{28}$ The compounds having 50-100 $\mu \mathrm{M}$ were mixed with $2.5 \mathrm{~mL}$ of phosphate buffer $(0.2 \mathrm{M}, \mathrm{pH} 6.6)$ and $2.5 \mathrm{~mL}$ of $1 \%$ potassium ferricyanide and incubated at $50{ }^{\circ} \mathrm{C}$ for $20 \mathrm{~min}$. To this mixture $2.5 \mathrm{~mL}$ of $10 \%$ trichloroacetic acid (TCA) was added and the mixture was centrifuged at $3000 \mathrm{rpm}$ for 20 min. The upper layer $(2.5 \mathrm{~mL})$ was mixed with $2.5 \mathrm{~mL}$ of deionised water and $0.5 \mathrm{~mL}$ of $0.1 \%$ Ferric chloride and the UV absorbance was measured at $700 \mathrm{~nm}$ using a spectro-

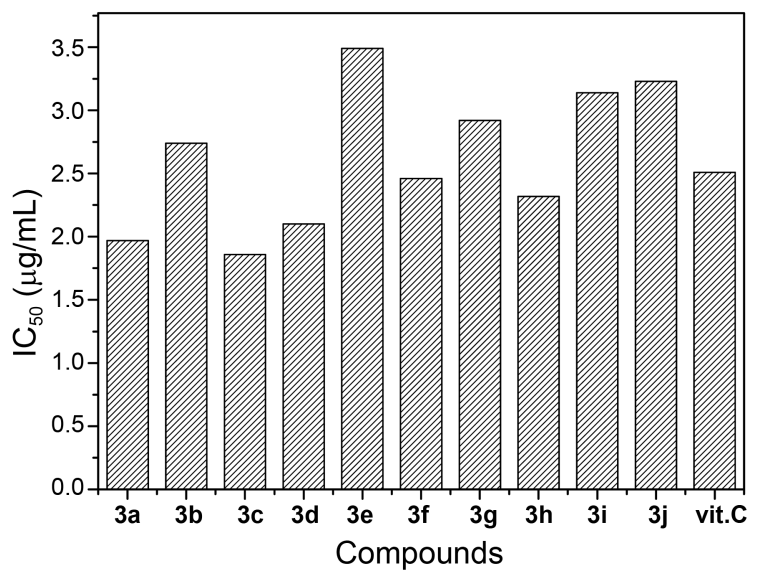

Figure 2. Reducing power assay.

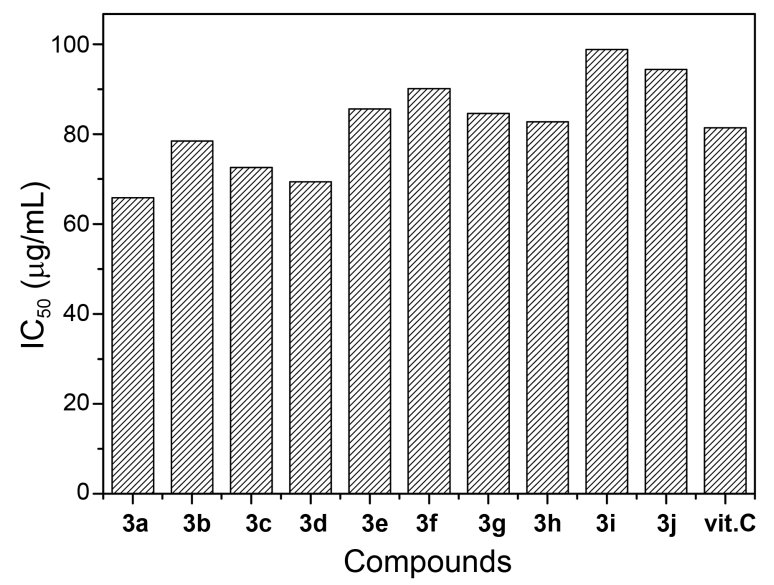

Figure 3. Lipid Peroxidation Assay.

photometer. Increase of absorbance of the reaction mixture indicate higher reducing power. Mean values from three independent samples were calculated for each compound and standard deviations were less than $5 \%$.

In the case of $\alpha$-hydroxyphosphonates 3a-j (Figure 2), derivatives $\mathbf{3 c}$ showed the highest reducing power with $\mathrm{IC}_{50}$ of $1.86 \mu \mathrm{g} / \mathrm{mL}$ when compared with other compounds. The remaining compounds exhibited reducing power activity in the following order: 3a ( $\left.\mathrm{IC}_{50} 1.97 \mu \mathrm{g} / \mathrm{mL}\right), \mathbf{3 d}\left(\mathrm{IC}_{50} 2.10 \mu \mathrm{g} /\right.$ $\mathrm{mL}), \mathbf{3 h}\left(\mathrm{IC}_{50} 2.32 \mu \mathrm{g} / \mathrm{mL}\right), \mathbf{3 f}\left(\mathrm{IC}_{50} 2.46 \mu \mathrm{g} / \mathrm{mL}\right), \mathbf{3 b}\left(\mathrm{IC}_{50}\right.$ $2.74 \mu \mathrm{g} / \mathrm{mL}), \mathbf{3 g}\left(\mathrm{IC}_{50} 2.92 \mu \mathrm{g} / \mathrm{mL}\right), 3 \mathbf{3 i}\left(\mathrm{IC}_{50} 3.14 \mu \mathrm{g} / \mathrm{mL}\right), \mathbf{3 j}$ $\left(\mathrm{IC}_{50} 3.23 \mu \mathrm{g} / \mathrm{mL}\right), 3 \mathbf{e}\left(\mathrm{IC}_{50} 3.49 \mu \mathrm{g} / \mathrm{mL}\right)$ and when compared with ascorbic acid ( $\left.\mathrm{IC}_{50} 2.51 \mu \mathrm{g} / \mathrm{mL}\right)$.

Lipid Peroxidation Assay. Lipid peroxidation was induced by $\mathrm{Fe}^{2+}$ ascorbate complex system in rat liver cells and was estimated as thiobarbituric acid reacting substances (TBARS) by the method of Ohkawa et al. ${ }^{29}$ Experiments in vitro lipid peroxidation were carried out to clarify the mode of the protective effect of the $\alpha$-hydroxyphosphonates against oxidative stress-induced cell damage. The inhibition of lipid peroxidation has been used as a model to elucidate antioxidant activity.

According to the results obtained (Figure 3), 3a ( $\mathrm{IC}_{50}$ $65.82 \mu \mathrm{g} / \mathrm{mL}$ ) significantly inhibited the ferric ion plus ascorbic acid in rat liver cells. The remaining compounds exhibited hydroxyl radical scavenging activity in the following order: 3d ( $\left.\mathrm{IC}_{50} 69.41 \mu \mathrm{g} / \mathrm{mL}\right), \mathbf{3 c}\left(\mathrm{IC}_{50} 72.57 \mu \mathrm{g} / \mathrm{mL}\right), \mathbf{3 b}$ (IC $\left.{ }_{50} 78.49 \mu \mathrm{g} / \mathrm{mL}\right), \mathbf{3 h}\left(\mathrm{IC}_{50} 82.74 \mu \mathrm{g} / \mathrm{mL}\right), \mathbf{3 g}\left(\mathrm{IC}_{50} 84.62\right.$ $\mu \mathrm{g} / \mathrm{mL}), \mathbf{3 e}\left(\mathrm{IC}_{50} 85.59 \mu \mathrm{g} / \mathrm{mL}\right), \mathbf{3 f}\left(\mathrm{IC}_{50} 90.12 \mu \mathrm{g} / \mathrm{mL}\right), \mathbf{3 j}$ ( $\left.\mathrm{IC}_{50} 94.36 \mu \mathrm{g} / \mathrm{mL}\right), 3 \mathbf{i}\left(\mathrm{IC}_{50} 98.79 \mu \mathrm{g} / \mathrm{mL}\right)$ and when compared with ascorbic acid $\left(\mathrm{IC}_{50} 81.4 \mu \mathrm{g} / \mathrm{mL}\right)$.

\section{Conclusion}

In conclusion, $\mathrm{KHSO}_{4}$ was found to be an efficient catalyst for the one-pot reaction of aldehyde and diethyl phosphite to afford the corresponding $\alpha$-hydroxyphosphonates in moderate to good yields. The main advantages of the present synthetic protocol are mild, solvent-free, ecofriendly catalyst and easy reaction work-up procedure. It is 
expected that the present methodology will find application in organic synthesis.

All the title compounds were screened for antioxidant properties by radical scavenging methods such as 1,1diphenyl-2-picryl hydrazyl (DPPH), Reducing power and lipid peroxidation. They exhibited potent in vitro antioxidant activity dose dependently.

Acknowledgments. The authors thank Prof. C.D. Reddy, Dept. of Chemistry, S.V. University, Tirupati for helpful discussions and for CSIR [01(2347)/09/EMR-II] New Delhi for providing financial assistance. The authors also express their thanks to Mr. P. Ramamohan, Dept. of Biochemistry, S.V. University, Tirupati for conducting antioxidant activity.

\section{References}

1. Schwetlick, K. In Mechanisms of Polymer of Degradation and Stabillisation; Elsevier Applied Science, London, 1990; p 23-26.

2. Schwetlick, K. Pure Appl. Chem. 1983, 55, 1629.

3. Tiku, M. L.; Liesch, J. B.; Robertson, F. M. J. Immunol. 1990, 145,690

4. Bax, B. E.; Alam, A. S.; Banerji, B.; Bax, C. M.; Bevis, P. J.; Stevens, C. R.; Moonga, B. S.; Blake, D. R.; Zaidi, M. Biochem. Biophys. Res. Commun. 1992, 183, 1153.

5. Bauerova, K.; Bezek, A. Gen. Physiol. Biophys. 1999, 18, 15.

6. Edmonds, S. E.; Blake, D. R.; Morris, C. J.; Winyard, P. G. J. Rheumatol. 1993, 37, 26.

7. (a) Dellaria, J. F.; Maki, R. G.; Stein, H. H.; Cohen, J.; Whittern, D.; Marsh, K.; Hoffman, D. J.; Plattner, J. J.; Perun, T. J. J. Med. Chem. 1990, 33, 534. (b) Bihovsky, R.; Tao, M.; Wells, G. J.; Mallamo, J. P. J. Med. Chem. 1998, 41, 3912.

8. Sikorski, J. A.; Miller, M. J.; Braccolino, D. S.; Cleary, D. G.; Corey, S. D.; Font, J. L.; Gruys, K. J.; Han, C. Y.; Lin, K. C.; Pansegrau, P. D.; Ream, J. E.; Schnur, D.; Shah, A.; Walker, M. C. Phosphorus, Sulfur and Silicon. 1993, 76, 375.

9. Pompliano, D. L.; Rands, E.; Schaber, M. D.; Mosser, S. D.; Anthony, N. J.; Gibbs, J. B. Biochemistry 1992, 31, 3800.

10. Stowasser, B.; Budt, K. H.; Li, J. Q.; Peyman, A.; Ruppert, D. Tetrahedron Lett. 1992, 33, 6625.

11. Snoeck, R.; Holy, A.; Dewolf-Peeters, C.; Van Den Oord, J.; De Clercq, E.; Andrei, G. Antimicrob. Agents Chemother. 2002, 46, 3356.

12. (a) Peters, M. L.; Leonard, M.; Licata, A. A. Clev. Clin. J. Med. 2001, 68, 945. (b) Z.Leder, B.; Kronenberg, H. M. Gastroenterology
2000, 119, 866 .

13. (a) Kafarski, P.; Lecjzak, B. J. Mol. Catal. B. 2004, 29, 99. (b) Maly, A.; Lecjzak, B.; Kafarski, P. Tetrahedron Asymm. 2003, 14, 1019.

14. (a) Wynberg, H.; Smaardijk, A. A. Tetrahedron Lett. 1983, 24, 5899. (b) Smaardijk, A. A.; Noorda, S.; Van Bolhuis, F.; Wynberg, H. Tetrahedron Lett. 1985, 26, 493.

15. (a) Akiyamam, T.; Morita, H.; Itoh, J.; Fuchibe, K. Org. Lett. 2005, 7, 2583. (b) Samanta, S.; Zhao, C. G. J. Am. Chem. Soc. 2006, 128,7442 .

16. Groaning, M. D.; Rowe, B. J.; Spilling, C. D. Tetrahedron Lett. 1998, 39, 5485.

17. (a) Zhou, X.; Liu, X.; Shang, D.; Xin, J.; Feng, X. Angew. Chem., Int. Ed. 2008, 47, 392. (b) Saito, B.; Katsuki, T. Angew. Chem., Int. Ed. 2005, 44, 4600.

18. (a) Duxbury, J. P.; Cawley, A.; Pett-Thornton, M.; Wantz, L.; Warne, J. N. D.; Greatrex, R.; Brown, D.; Kee, T. P. Tetrahedron Lett. 1999, 40, 4403. (b) Duxbury, J. P.; Warne, J. N. D.; Mushtaq, R.; Ward, W.; Pett-Thornton, M.; Jiang, M.; Greatrex, R.; Kee, T. P. Organometallics. 2000, 19, 4445.

19. Arai, T.; Bougauchi, M.; Sasai, H.; Shibaski, M. J. Org. Chem. 1996, 61, 2926.

20. (a) Texier-Boullet, F.; Lequitte, M. Tetrahedron Lett. 1986, 27, 3515. (b) Villemin, D.; Racha, R. Tetrahedron Lett. 1986, 27, 1789.

21. Swapnil, S. S.; Amol, H. K.; Madhav, N. W.; Charansingh, H. G.; Bapurao, B. S.; Murlidhar, S. S. Arkivoc. 2009, ii, 138.

22. (a) Simoni, D.; Rondanin, R.; Morini, M.; Baruchello, R.; Lnvidiata, F. P. Tetrahedron Lett. 2000, 41, 1607. (b) Alexander Christopher, W.; Philip Albiniak, A.; Lisa Gibson, R. Phosphorus, Sulfur and Silicon Relat. Elem. 2000, 167, 205.

23. (a) Kumar, R. S.; Nagarajan, R.; Perumal, P. T. Synthesis 2004, 6, 949. (b) Nagarajan, R.; Perumal, P. T. Chem. Lett. 2004, 33, 288.

24. (a) Chandra Sekhar Reddy, G.; Veera Narayana Reddy, M.; Bakthavatchala Reddy, N.; Suresh Reddy, C. Phosphorus, Sulfur and Siliion. 2011, 186, 74. (b) Veera Narayana Reddy, M.; Bala Krishna, A.; Suresh Reddy, C. Eur. J. of Med.Chem. 2010, 45, 1828.

25. Tajbakhsh, Md.; Heydari, A.; Khalilzadeh, M. A.; Lakouraj, M. M.; Zamenian, B.; Khaksar, S. Synlett. 2007, 15, 2347.

26. Veera Narayana Reddy, M.; Balakrishna, A.; Anil Kumar, M.; Chandra Sekhar Reddy, G.; Uma Ravi Sankar, A.; Suresh Reddy, C.; Murali Krishna, T. Chem. Pharm. Bull. 2009, 57, 1391.

27. Choi, C. W.; Kim, S. C.; Hwang, S. S.; choi, B. K.; Ahn, H. J.; Lee, M. Y.; Park, S. H.; Kim, S. K. Plant Sci. 2002, 153, 1161.

28. Oyaizu, M. Jpn. J. Nutr. 1986, 44, 307.

29. Ohkawa, N.; Ohishi, K.; Yagi, K. Anal. Biochem. 1979, 95, 351. 